\title{
Interaction of Atomic Nitrogen with Electronically Excited Molecular Oxygen: a Theoretical Study
}

\author{
A.V. Pelevkin ${ }^{1,2}$, I.N. Kadochnikov ${ }^{1}$, A.S. Sharipov ${ }^{1}$ \\ ${ }^{1}$ Central Institute of Aviation Motors, Moscow, 111116, Russian Federation \\ ${ }^{2}$ Moscow Institute of Physics and Technology, \\ Dolgoprudny, Moscow Region, 141700, Russian Federation \\ pelevkin@phystech.edu
}

\begin{abstract}
Under conditions of violation of the thermodynamic equilibrium between the internal degrees of freedom of particles, the kinetics of the ongoing physicochemical processes can differ significantly from the equilibrium case. One of the important reactions responsible for the formation of nitrogen oxides in air is the reaction of a nitrogen atom with an oxygen molecule, which can be in the ground state as well as in the electronically excited states under the influence of a nonequilibrium electric discharge, resonant laser radiation or behind the front of a strong shock wave. Quantum chemical calculations were performed using the extended multi-configuration quasi-degenerate perturbation theory to study the reaction of the interaction of a nitrogen atom with an electronically excited molecule $\mathrm{O}_{2}$ molecule in the electronic states $a^{1} \Delta_{g}$ and $b^{1} \Sigma_{g}^{+}$, quantum-chemical calculations were performed using the extended multi-configuration quasidegenerate second-order perturbation theory. Energetically favorable reaction paths, minimum energy crossing points, and the corresponding quenching channels were found. It was shown that the activation barrier of the reaction $\mathrm{N}\left({ }^{4} S\right)+\mathrm{O}_{2}\left(a^{1} \Delta g\right)$ is 2.3 times larger than the barrier of the reaction of a nitrogen atom with $\mathrm{O}_{2}$ in the ground state, however, for an accurate estimate of the constant of this process, it is also necessary to take into account the probability of a nonadiabatic transition $\mathrm{N}\left({ }^{4} S\right)+\mathrm{O}_{2}\left(a^{1} \Delta g\right) \rightarrow \mathrm{N}\left({ }^{4} S\right)+\mathrm{O}_{2}\left(X^{3} \Sigma_{g}{ }^{-}\right)$. It was shown for the first time that the reaction of atomic nitrogen and $\mathrm{O}_{2}\left(b^{1} \Sigma_{g}^{+}\right)$leads to the formation of a molecule $\mathrm{NO}\left(\mathrm{X}^{2} \Pi_{r}\right)$ and an atom $\mathrm{O}\left({ }^{3} P\right)$. Besides, the quenching process $\mathrm{N}\left({ }^{4} S\right)+\mathrm{O}_{2}\left(b^{1} \Sigma_{g}{ }^{+}\right) \rightarrow \mathrm{N}\left({ }^{2} D\right)+\mathrm{O}_{2}\left(X^{3} \Sigma_{g}{ }^{-}\right)$is also possible. In the future, the rate constants of these processes will be estimated within the variational and non-adiabatic transition state theories.
\end{abstract}

Keywords: nitrogen, oxygen, nonequilibrium discharge plasma, electron excitation, quantum chemistry.

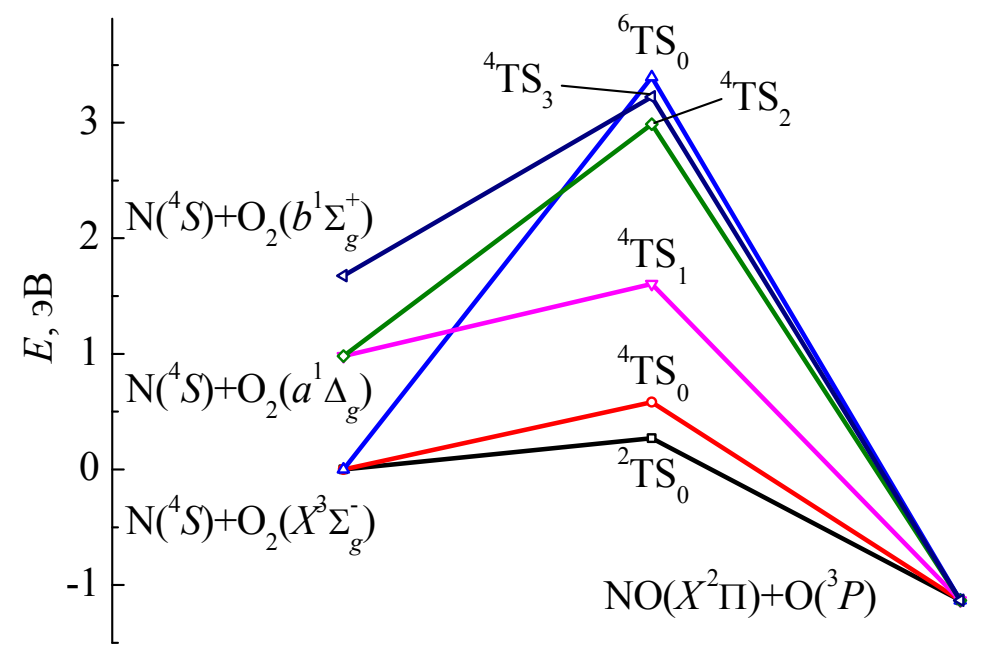

Energy diagram of system $\mathrm{N}+\mathrm{O}_{2}$ 


\title{
Теоретическое исследование взаимодействия атомарного азота с электронно-возбужденным молекулярным кислородом
}

\author{
А.В. Пелевкин ${ }^{1,2}$, И.Н. Кадочников ${ }^{1}$, А.С. Шарипов ${ }^{1}$ \\ ${ }^{1}$ ЦИАМ им. П.И. Баранова, Россия, Москва, 111116, ул. Авиамоторная, 2 \\ ${ }^{2}$ МФТИ (НИУ), Россия, Долгопрудный, 141701, Институтский пер., 9 \\ pelevkin@phystech.edu
}

\begin{abstract}
Аннотация
В условиях нарушения термодинамического равновесия между внутренними степенями свободы частиц кинетика протекающих физико-химических процессов может существенно отличаться от равновесного случая. Одной из важных реакций, ответственных за образование оксидов азота в воздухе, является реакция атома азота с молекулой кислорода, который в условиях воздействия неравновесного электрического разряда, резонансного лазерного излучения или за фронтом сильной ударной волны может находиться не только в основном, но и в электронно-возбужденных состояниях. Для изучения реакции взаимодействия атома азота с электронно-возбужденной молекулой $\mathrm{O}_{2}$ в электронных состояниях $a^{1} \Delta g$ и $b^{1} \Sigma_{g}^{+}$проведены квантово-химические расчеты с использованием расширенной многоконфигурационной квазивырожденной теории возмущения второго порядка. Были обнаружены энергетически выгодные пути реакции, а также точки межсистемных переходов с минимальной энергией и соответствующие им каналы тушения. Показано, что активационный барьер реакции $\mathrm{N}\left({ }^{4} S\right)+\mathrm{O}_{2}\left(a^{1} \Delta g\right)$ больше, чем барьер реакции атома азота с $\mathrm{O}_{2}$ в основном состоянии в 2.3 раза, однако для точной оценки константы данного процесса необходимо также учитывать вероятность неадиабатического перехода $\mathrm{N}\left({ }^{4} S\right)+\mathrm{O}_{2}\left(a^{1} \Delta g\right) \rightarrow \mathrm{N}\left({ }^{4} S\right)+\mathrm{O}_{2}\left(X^{3} \Sigma_{g}{ }^{-}\right)$. Впервые было показано, что реакция атомарного азота и $\mathrm{O}_{2}\left(b^{1} \Sigma_{g}^{+}\right)$приводит к образованию молекулы $\mathrm{NO}\left(X^{2} \Pi_{r}\right)$ и атома $\mathrm{O}\left({ }^{3} P\right)$. Помимо этого, также возможен процесс тушения $\mathrm{N}\left({ }^{4} S\right)+\mathrm{O}_{2}\left(b^{1} \Sigma_{g}{ }^{+}\right) \rightarrow \mathrm{N}\left({ }^{2} D\right)+\mathrm{O}_{2}\left(X^{3} \Sigma_{g}{ }^{-}\right)$. В дальнейшем планируется провести оценку констант скорости данных процессов в рамках вариационной и неадиабатической теорий переходного состояния.
\end{abstract}

Ключевые слова: азот, кислород, неравновесная разрядная плазма, электронное возбуждение, квантовая химия.

\section{1. Введение}

Применение плазмы неравновесного разряда, содержащей высокоактивные частицы (атомы, ионы, радикалы, колебательно- и электронно-возбужденные молекулы), для воспламенения и управления процессами горения (так называемое плазменно-стимулированное горение) является одним из перспективных способов воздействия на горючую смесь за счет потенциально более высокой энергетической эффективности по сравнению с другими способами [1-8]. Однако неизвестно, каким образом при плазменно-стимулированном горении наличие тех или иных частиц с высокой реакционной способностью может в конечном итоге повлиять на образование $\mathrm{NO}_{x}$ на выходе из двигателя. Также анализ данной проблемы осложняется тем, что кинетика процессов образования оксидов азота в условиях плазменно-индуцированного горения в воздухе остается малоизученной $[1,2,9]$.

Ключевым параметром, который определяет процессы образования активных частиц и энерговыделение в плазме газового разряда, является величина приведенного электрического 
поля $E / N$ ( $E$ - напряженность электрического поля, $N$ - концентрация частиц). Многочисленные исследования проводились в различное время для электрических разрядов в газе преимущественно при высоких значениях $E / N$ (различные модификации высоковольтных наносекундных и диэлектрических барьерных разрядов) $[1,2,4,8]$, когда энергия разряда идет в основном на диссоциацию и ионизацию молекулярных компонентов топлива и воздуха [10]. В этом случае для моделирования окисления топлива и сопутствующего образования оксидов азота [9] могут быть использованы термически равновесные кинетические схемы.

В то же время при достаточно низких значениях $E / N$, когда происходит эффективное возбуждение электронных состояний молекулярного кислорода $[1,2,7]$, имеет место существенно иная ситуация. В частности, возбуждение $\mathrm{O}_{2}$ в различные электронные состояния $\left(a^{1} \Delta g, b^{1} \Sigma_{g}^{+}\right.$и т.д.) с помощью тлеющего электрического разряда предполагается критически важным в смысле энергоэффективной интенсификации цепных процессов в различных горючих смесях [11-13]. Отметим также, что электронное возбуждение $\mathrm{O}_{2}$ представляет интерес и с точки зрения лазерно-индуцированного горения [14-15], которое достигается за счет воздействия на газовую смесь резонансного лазерного излучения. В этой связи для предсказательного моделирования кинетики образования $\mathrm{NO}_{x}$ требуется детальная кинетическая информация об основных процессах с участием электронно-возбужденных молекул $\mathrm{O}_{2}$, участвующих в образовании оксидов азота.

Образование синглетного кислорода $\mathrm{O}_{2}\left(a^{1} \Delta g\right)$ также происходит за фронтом сильной ударной волны в воздухе за счет Е-Т релаксации и химических превращений, что может влиять на цепные процессы в горючей смеси за фронтом ударной волны, а также давать вклад в оптическую плотность среды (оптические шлирен- и теневые методы могут применяться для диагностики термической неравновесности в газовых потоках, см. [16]). Так, ранее была построена уровневая кинетическая модель для воздушной плазмы, учитывающая широкий набор химических и плазмохимических реакций с участием молекул как в основном, так и в возбуждённых электронных состояниях [17]. В рамках этой модели были проведены расчёты типичных параметров неравновесного газа за фронтом сильной ударной волны и выделены процессы, оказывающие наиболее существенное влияние на изменение компонентного состава воздушной плазмы. Расчет показал, что электронно-возбуждённые молекулы $\mathrm{N}_{2}, \mathrm{NO}$ и $\mathrm{N}_{2}{ }^{+}$образуются вследствие протекания процессов Е-Т релаксации, однако образование электронно-возбуждённых молекул кислорода происходит по другому механизму. На рис. 1 представлены результаты одного из расчетов по этой модели. Видно, что Е-Т релаксация не приводит к существенному возбуждению электронных состояний ввиду быстрого распада молекул $\mathrm{O}_{2}\left(X^{3} \Sigma_{g}^{-}\right)$в релаксационной зоне. Согласно тем же расчётам, возбуждение электронного состояния $a^{1} \Delta g$ молекул $\mathrm{O}_{2}$ за сильной ударной волной происходит преимущественно вследствие реакции $\mathrm{N}\left({ }^{4} S\right)+\mathrm{O}_{2}\left(a^{1} \Delta_{g}\right)=\mathrm{O}\left({ }^{3} P\right)+\mathrm{NO}\left(X^{2} \Pi_{r}\right)$, протекающей в обратном направлении. При этом важно отметить, что константа скорости данного процесса была получена на основе энергетического барьера, определенного методом CASSCF без учета динамической корреляции [9], что, как правило, существенным образом сказывается на величине получаемой константы. Поэтому целесообразно учесть эффект динамической электронной корреляции на динамику этой реакции, а также реакции $\mathrm{N}\left({ }^{4} S\right)+\mathrm{O}_{2}\left(b^{1} \Sigma_{g}^{+}\right)=\mathrm{O}\left({ }^{3} P\right)+\mathrm{NO}\left(X^{2} \Pi_{r}\right)$, для которой константа скорости достоверно не определена. Стоит отметить, что данные реакции важны не только для учета возбуждения синглетного кислорода, но и для уточнения кинетической модели для азотной (воздушной) плазмы в целом [17].

Из всего вышесказанного следует необходимость в подробном изучении кинетики элементарных процессов в системе $\mathrm{N}+\mathrm{O}_{2}\left(\left\{a^{1} \Delta_{g}, b^{1} \Sigma_{g}^{+}\right\}\right)$с использованием многоконфигурационных методов ab initio. 


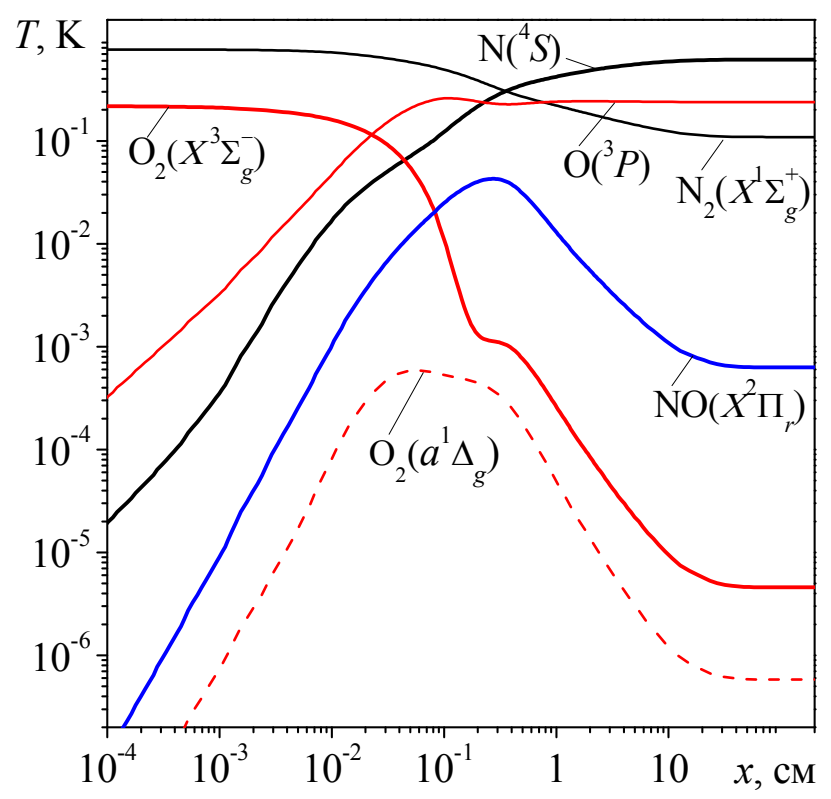

Рис. 1. Изменение мольных долей компонентов азотной плазмы в основном электронном состоянии $\mathrm{N}_{2}\left(X^{1} \Sigma_{g}^{+}\right), \mathrm{N}\left({ }^{4} S\right), \mathrm{O}_{2}\left(X^{3} \Sigma_{g}\right), \mathrm{O}\left({ }^{3} P\right), \mathrm{NO}\left(X^{2} \Pi_{r}\right)$, а также электронно-возбуждённых молекул $\mathrm{O}_{2}\left(a^{1} \Delta g\right)$ в потоке за фронтом сильной ударной волны при начальных условиях перед ударной волной $T_{0}=300 \mathrm{~K}$, $P_{0}=0.1$ торр и $u_{0}=8$ км $/ \mathrm{c}$, определённое с использованием уровневой кинетической модели [17]

\section{2. Методология исследования: исследование поверхности потенциальной энергии}

Для подробного исследования поверхности потенциальной энергии (ППЭ) системы $\mathrm{N}+\mathrm{O}_{2}\left(\left\{X^{3} \Sigma_{\bar{g}}, a^{1} \Delta_{g}, b^{1} \Sigma_{g}^{+}\right\}\right)$были проведены квантово-химические расчеты с использованием метода расширенной многоконфигурационной квазивырожденной теории возмущения второго порядка (XMCQDPT2) [18]. Для данного метода в качестве нулевого приближения волновой функции была взята волновая функция, полученная методом усредненного по состояниям многоконфигурационного самосогласованного поля в полном активном пространстве (SA-CASSCF) [19] с равными весами для рассматриваемых состояний. Для данной системы был выбран размер активного пространства в 11 электронов, находящихся на 9 орбиталях (сокращенно $(11,9))$. В такое активное пространство включены все р-электроны атома $\mathrm{N}$ и двух атомов О. Во всех расчетах в качестве основного базисного набора было взято корреляционносогласованное семейство базисов Даннинга с диффузными функциями (aug-cc-p $\mathrm{V} X \mathrm{Z}$, $X=2 \div 4$ ) [20]. Все квантово-химические расчеты были проведены в программном пакете Firefly v. 8.2.0 [21], частично основанном на исходном коде пакета GAMESS (US)[22].

Для рассматриваемых термов структуры реагентов, переходных состояний (TS) и возможных продуктов реакций были оптимизированы на уровне теории XMCQDPT2(11,9)/augcc-pVDZ. Для данных структур на том же уровне теории был проведен анализ гармонических частот колебаний для проверки количества мнимых частот и расчета энергии нулевых колебаний (ЭНК). Для каждого выявленного переходного состояния TS был построен путь минимальной энергии реакции (путь МЭР) методом внутренней координаты реакции ГонзалесаШлегеля [23] (на том же уровне теории). Для поиска возможных точек межсистемных переходов (IC) вдоль выявленных путей МЭР были построены их проекции на соседние термы.

Для поиска точек межсистемных переходов с минимальной энергией (Minimum Energy Crossing Point, MECP) была проведена оптимизация структур IC с использованием метода множителей Лагранжа [24] на уровне теории XMCQDPT2(11,9)/aug-cc-pVDZ. 


\section{3. Обсуждение результатов}

\section{1. Расчеты электронных термов}

На рис. 2 представлена диаграмма энергий для трех низших электронных состояний системы $\mathrm{N}+\mathrm{O}_{2}$, полученная в ходе многоконфигурационных расчетов. Здесь и далее мы ввели следующие обозначения для критических точек (TS): левый верхний индекс обозначает спиновую мультиплетность, а правый нижний индекс - порядковый номер данной критической точки среди этих точек той же мультиплетности. Отметим также, что при расчете структуры и энергии реагентов и продуктов отдельные частицы располагались на расстоянии около $4 \AA$.

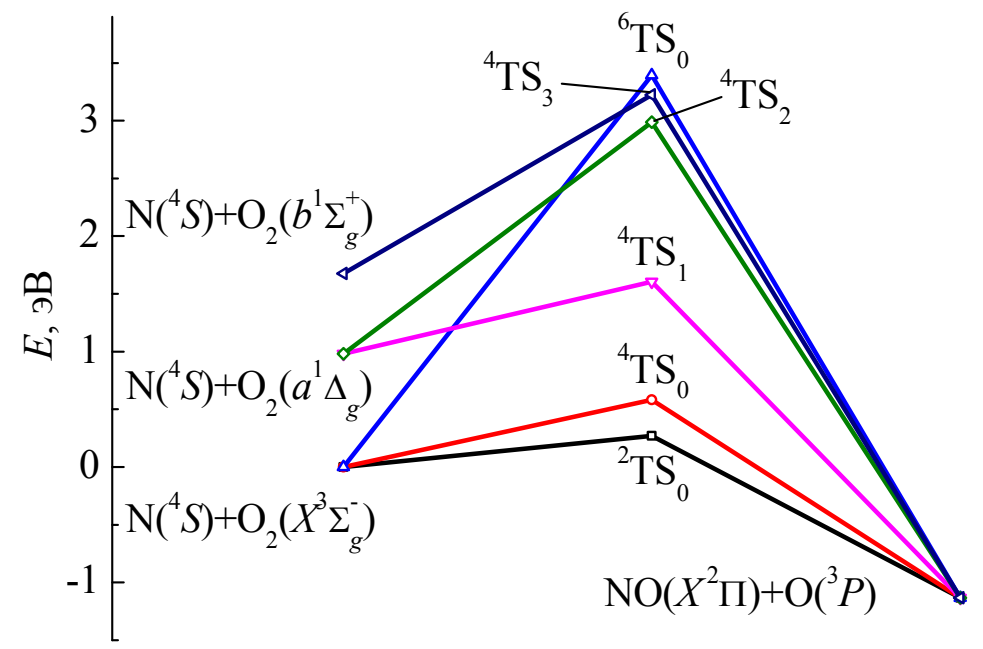

Рис. 2. Диаграмма энергии системы $\mathrm{N}+\mathrm{O}_{2}$. Значения энергии были получены на уровне теории XMCQDPT2/aug-cc-pVQZ // XMCQDPT2/aug-cc-pVDZ c учетом энергии нулевых колебаний

В табл. 1 приведены значения энергии возбуждения $T_{\mathrm{e}}$ для электронных состояний молекул $\mathrm{O}_{2}$, полученные в рамках методологии данной работы, в сравнении с эталонными спектроскопическими измерениями [25]. Можно сделать вывод, что использование метода возмущений второго порядка позволяет в пределах выбранного активного пространства $(11,9)$ получить оценки энергии $T_{\mathrm{e}}$ в разумном согласии со спектроскопическими наблюдениями.

\section{Таблица 1}

Значения $T_{\mathrm{e}}$, полученные с использованием методологии настоящей работы в сравнении со спектроскопическими данными [25] (в эВ)

\begin{tabular}{|c|c|c|}
\hline состояние & наша методология & справ. данные \\
\hline $\mathrm{O}_{2}\left(a^{1} \Delta_{g}\right)$ & 0.981 & $0.976[25]$ \\
\hline $\mathrm{O}_{2}\left(b^{1} \Sigma_{g}^{+}\right)$ & 1.676 & $1.627[25]$ \\
\hline
\end{tabular}

Константа скорости реакции атомарного азота $\mathrm{N}\left({ }^{4} S\right)$ с молекулярным кислородом в основном состоянии $\mathrm{O}_{2}\left(X^{3} \Sigma_{g}\right)$ была определена ранее с достаточно высокой точностью и находится в хорошем согласии с экспериментальными данными $[9,26]$. По этой причине в дальнейшем не планируется проводить оценку константы скорости этой реакции.

Что касается системы $\mathrm{N}+\mathrm{O}_{2}\left(a^{1} \Delta_{g}\right)$, то из-за двукратного вырождения молекулы $\mathrm{O}_{2}\left(a^{1} \Delta_{g}\right)$ её взаимодействие с атомом $\mathrm{N}\left({ }^{4} S\right)$ протекает вдоль двух разных ППЭ через переходные состояния ${ }^{4} \mathrm{TS}_{1}$ (путь реакции $\left.(\mathrm{d})\right)$ и ${ }^{4} \mathrm{TS}_{2}$ (путь реакции (e)) с разной энергией активации (см. рис. 1). Движение системы вдоль соответствующих путей минимальной энергии реакции приводит к образованию $\mathrm{NO}\left(X^{2} \Pi_{r}\right)$ и $\mathrm{O}\left({ }^{3} P\right)$. Стоит отметить, что в прошлых 
работах [9] путь реакции (е) не был обнаружен, несмотря на двукратное электронное вырождение системы $\mathrm{N}+\mathrm{O}_{2}\left(a^{1} \Delta_{g}\right)$.

В системе $\mathrm{N}+\mathrm{O}_{2}\left(b^{1} \Sigma_{g}^{+}\right)$взаимодействие реагентов протекает через переходное состояние ${ }^{4} \mathrm{TS}_{3}$ (путь реакции (f)) с довольно высоким энергетическим барьером. Продуктами этой реакции, как и в системе $\mathrm{N}+\mathrm{O}_{2}\left(a^{1} \Delta_{g}\right)$, так же являются $\mathrm{NO}\left(X^{2} \Pi_{r}\right)$ и $\mathrm{O}\left({ }^{3} P\right)$.

Обнаруженные пути реакции в ходе исследования поверхности потенциальной энергии системы $\mathrm{N}+\mathrm{O}_{2}\left(\left\{X^{3} \Sigma_{g}^{-}, a^{1} \Delta_{g}, b^{1} \Sigma_{g}^{+}\right\}\right)$, а также энергии активации в прямом и обратном направлениях и энтальпии реакции приведены в табл. 2 . Все выявленные реакции оказались экзотермическими $\left(\left|\Delta_{r} H^{\circ}\right|>1\right.$ эВ). Стоит отметить, что полученное значение энтальпии реакции (a) $(-1.133$ эВ) довольно близко к значению, взятому из Active Thermochemical Tables [27] $(-1.380$ эВ).

\section{Таблииа 2}

Выявленные пути реакции и соответствующие им значения энергии активации в прямом и обратном направлениях $\left(E_{\mathrm{a}}^{+}\right.$и $\left.E_{\mathrm{a}}{ }^{-}\right)$и энтальпия реакции $\left(\Delta_{\mathrm{r}} H^{0}\right)($ в эВ)

\begin{tabular}{|c|c|c|c|c|}
\hline обознач. & путь реакции & $E_{\mathrm{a}}^{+}$ & $E_{\mathrm{a}}^{-}$ & $\Delta_{r} H^{\circ}$ \\
\hline (a) & $\mathrm{N}\left({ }^{4} S\right)+\mathrm{O}_{2}\left(X^{3} \Sigma_{g}^{-}\right) \rightarrow \mathrm{NO}\left(X^{2} \Pi_{r}\right)+\mathrm{O}\left({ }^{3} P\right)$ & 0.271 & 1.404 & -1.133 \\
\hline (b) & $\mathrm{N}\left({ }^{4} S\right)+\mathrm{O}_{2}\left(X^{3} \Sigma_{g}^{-}\right) \rightarrow \mathrm{NO}\left(X^{2} \Pi_{r}\right)+\mathrm{O}\left({ }^{3} P\right)$ & 0.582 & 1.715 & -1.133 \\
\hline (c) & $\mathrm{N}\left({ }^{4} S\right)+\mathrm{O}_{2}\left(X^{3} \Sigma_{g}^{-}\right) \rightarrow \mathrm{NO}\left(X^{2} \Pi_{r}\right)+\mathrm{O}\left({ }^{3} P\right)$ & 3.395 & 4.528 & -1.133 \\
\hline (d) & $\mathrm{N}\left({ }^{4} S\right)+\mathrm{O}_{2}\left(a^{1} \Delta_{g}\right) \rightarrow \mathrm{NO}\left(X^{2} \Pi_{r}\right)+\mathrm{O}\left({ }^{3} P\right)$ & 0.625 & 2.740 & -2.114 \\
\hline (e) & $\mathrm{N}\left({ }^{4} S\right)+\mathrm{O}_{2}\left(a^{1} \Delta_{g}\right) \rightarrow \mathrm{NO}\left(X^{2} \Pi_{r}\right)+\mathrm{O}\left({ }^{3} P\right)$ & 2.007 & 4.121 & -2.114 \\
\hline (f) & $\mathrm{N}\left({ }^{4} S\right)+\mathrm{O}_{2}\left(b^{1} \Sigma_{g}^{+}\right) \rightarrow \mathrm{NO}\left(X^{2} \Pi_{r}\right)+\mathrm{O}\left({ }^{3} P\right)$ & 1.548 & 4.357 & -2.809 \\
\hline
\end{tabular}

Полученное значение $E_{\mathrm{a}}^{+}$для пути (d) оказалось примерно в 2.2 раза меньше, чем в одной из прошлых работ $\left(E_{\mathrm{a}}^{+}=1.417 \mathrm{\jmath B}\right)[9]$. Такая существенная разница в оценке активационного барьера связана с тем, что в работе [9] в расчетах ab initio был применен метод CASSCF, который не учитывает динамическую энергию корреляции. Именно для учета этой части энергии в настоящей работе был использован метод расширенной многоконфигурационной квазивырожденной теории возмущения второго порядка XMCQDPT2 [18]. Также одним из существенных отличий в методологиях данных работ, что может привести к некоторой разнице в результатах, является размер выбранного активного пространства: в настоящей работе он подробнее - (11.9) против (9.7) в [9].

\section{2. Поиск возможных межсистемных переходов}

Для обнаружения возможных межсистемных переходов были построены пути минимальной энергии реакций (d)-(f), а также их проекции на соседние ППЭ. На рис. 3 приведены результаты данного построения. На рис. 3 видно, что для пути (d) в окрестности переходного состояния ${ }^{4} \mathrm{TS}_{1}$ наблюдается пересечение с термом $\mathrm{N}\left({ }^{4} S\right)+\mathrm{O}_{2}\left(X^{3} \Sigma_{g}^{-}\right)$(данный терм характеризуется мультиплетностью $2 S+1=6$ ), что не было учтено в прошлых работах [9] при оценке константы скорости реакции (d). Другие межсистемные пересечения на пути (d), находящиеся за переходным состоянием ${ }^{4} \mathrm{TS}_{1}$, не имеют химического значения, поскольку в этой области пересекающиеся термы спускаются к одним и тем же продуктам - $\operatorname{NO}\left(X^{2} \Pi_{r}\right)$ и $\mathrm{O}\left({ }^{3} P\right)$ (см. рис. $\left.3, a\right)$.

Что касается второго терма системы $\mathrm{N}+\mathrm{O}_{2}\left(a^{1} \Delta_{g}\right)$, на рис. $3, \sigma$ видно, что путь реакции (е) пересекается с двумя термами до прохождения переходного состояния ${ }^{4} \mathrm{TS}_{2}-\mathrm{c}$ одним термом высоколежащей системы $\mathrm{N}\left({ }^{2} D\right)+\mathrm{O}_{2}\left(X^{3} \Sigma_{\bar{g}}\right)$ мультиплетности $2 S+1=2$ и с одним термом $\mathrm{N}\left({ }^{4} S\right)+\mathrm{O}_{2}\left(X^{3} \Sigma_{\bar{g}}\right)$ мультиплетности $2 S+1=6$. Как бы то ни было, активационный барьер пути реакции (e) в три раза выше, чем барьер пути реакции (d), поэтому при оценке 
константы скорости данный путь можно не учитывать. По аналогичным соображениям можно пренебречь возможными межсистемными переходами, поскольку они расположены значительно выше, чем соответствующие переходы вдоль пути реакции (d).

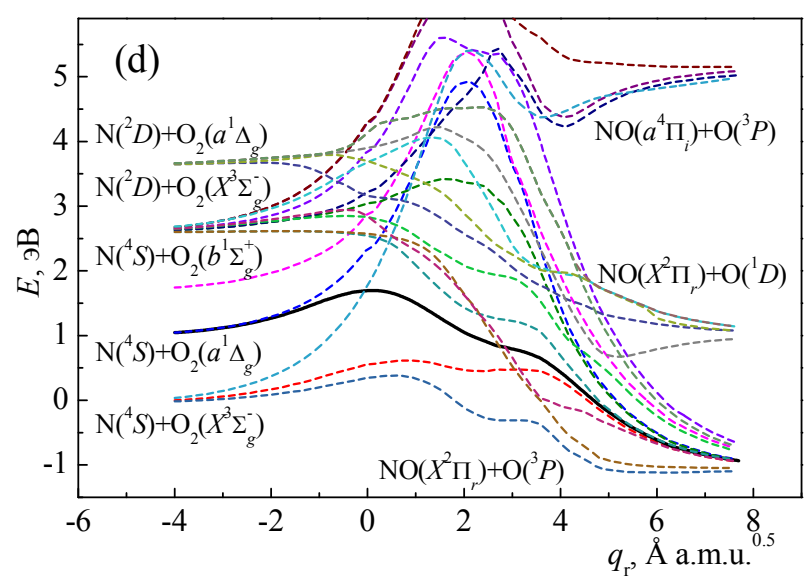

$a$

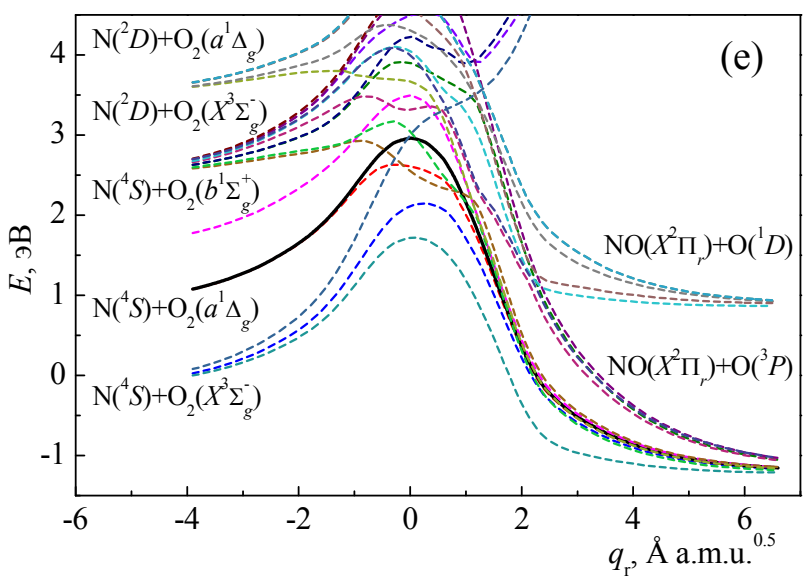

$\sigma$

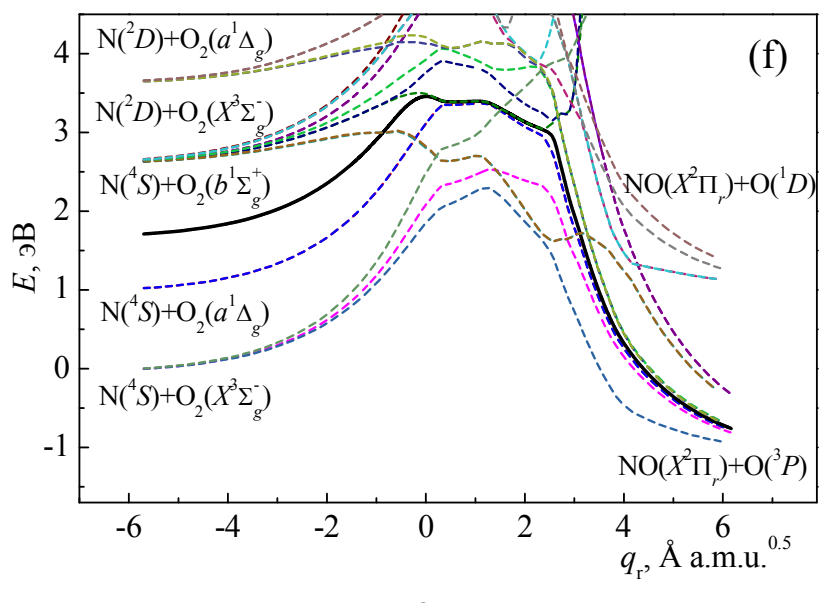

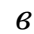

Рис. 3. Профили энергии в зависимости от внутренней координаты (сплошная черная кривая) и их проекции на соседние ППЭ (пунктирные кривые): $a$ ) для пути реакции (d); б) для пути реакции (e); в) для пути реакции (f)

Как видно по рис. 3, в, до преодоления переходного состояния ${ }^{4} \mathrm{TS}_{3}$ терм $\mathrm{N}\left({ }^{4} S\right)+\mathrm{O}_{2}\left(b^{1} \Sigma_{g}^{+}\right)$пересекается с двумя термами $\mathrm{N}\left({ }^{2} D\right)+\mathrm{O}_{2}\left(X^{3} \Sigma_{g}^{-}\right)$близкой энергии и мультиплетности $2 S+1=2$, а после прохождения ${ }^{4} \mathrm{TS}_{3}-$ с термом $\mathrm{N}\left({ }^{4} S\right)+\mathrm{O}_{2}\left(X^{3} \Sigma_{\bar{g}}\right)$ мультиплетности $2 S+1=6$. Дальнейшие пересечения терма $\mathrm{N}\left({ }^{4} S\right)+\mathrm{O}_{2}\left(b^{1} \Sigma_{g}^{+}\right)$, как и в случае с рассмотренными выше системами, можно не учитывать при оценке константы скорости.

Для поиска МЕСР проводилась процедура оптимизации в рамках методологии данной работы, а в качестве начальной точки принимались точки межсистемного перехода на пути реакции (d) и (f).

\section{3. Кинетика реакций}

На основе проведенных исследований системы $\mathrm{N}+\mathrm{O}_{2}\left(\left\{X^{3} \Sigma_{g}, a^{1} \Delta_{g}, b^{1} \Sigma_{g}^{+}\right\}\right)$можно выделить следующие реакционные каналы $\left(R_{i}\right)$ :

$$
\begin{aligned}
& \mathrm{N}\left({ }^{4} S\right)+\mathrm{O}_{2}\left(X^{3} \Sigma_{g}^{-}\right) \rightarrow \mathrm{NO}\left(X^{2} \Pi_{r}\right)+\mathrm{O}\left({ }^{3} P\right), \\
& \mathrm{N}\left({ }^{4} S\right)+\mathrm{O}_{2}\left(a^{1} \Delta_{g}\right) \rightarrow \mathrm{NO}\left(X^{2} \Pi_{r}\right)+\mathrm{O}\left({ }^{3} P\right), \\
& \mathrm{N}\left({ }^{4} S\right)+\mathrm{O}_{2}\left(b^{1} \Sigma_{g}^{+}\right) \rightarrow \mathrm{NO}\left(X^{2} \Pi_{r}\right)+\mathrm{O}\left({ }^{3} P\right),
\end{aligned}
$$


а также каналы тушения $\left(\mathrm{Q}_{i}\right)$ :

$$
\begin{aligned}
& \mathrm{N}\left({ }^{4} S\right)+\mathrm{O}_{2}\left(a^{1} \Delta_{g}\right) \rightarrow \mathrm{N}\left({ }^{4} S\right)+\mathrm{O}_{2}\left(X^{3} \Sigma_{g}^{-}\right), \\
& \mathrm{N}\left({ }^{4} S\right)+\mathrm{O}_{2}\left(b^{1} \Sigma_{g}^{+}\right) \rightarrow \mathrm{N}\left({ }^{2} D\right)+\mathrm{O}_{2}\left(X^{3} \Sigma_{g}^{-}\right)
\end{aligned}
$$

Как уже было отмечено выше, оценка константы процесса (R1) была проведена ранее с достаточной степенью точности (см. [9]). Однако для реакции (R2) при оценке константы скорости необходимо учитывать вероятность межсистемного перехода из-за пересечения с термом $\mathrm{N}\left({ }^{4} S\right)+\mathrm{O}_{2}\left(X^{3} \Sigma_{g}^{-}\right)$. Тем не менее, до сих пор данный факт не учитывался ранее, в том числе и в [9]. Стоит также отметить, что при оценке константы скорости (R2) на основе результатов, полученных в настоящей работе, можно ожидать увеличение значений константы по сравнению с результатами работы [9]. Связано это с существенной разницей в энергии активации реакции (R2) - в нашей работе это значение в 2.2 раза меньше, чем в [9]. Однако с применением невариационной теории переходного состояния, а также учетом вероятности неадиабатического перехода увеличение эффективной константы скорости реакции (R2) может оказаться не столь существенным. Также из-за наличия обозначенного выше межсистемного пересечения следует возможность существования канала тушения (Q1), а после локализации соответствующей точки МЕСР возможно будет провести оценку константы скорости данного процесса в рамках неадиабатической теории переходного состояния.

Что касается реакции (R3), то константу скорости данного процесса оценить довольно затруднительно из-за множества пересечений с различными термами. Тем не менее, на основе неадиабатической теории переходного состояния возможна теоретическая оценка процесса тушения (Q2), энергетический барьер которого ниже, чем у реакционного канала (R3).

Таким образом, на основе полученных результатов в будущем планируется провести оценку констант скорости для следующих реакций:

$$
\begin{aligned}
& \mathrm{N}\left({ }^{4} S\right)+\mathrm{O}_{2}\left(a^{1} \Delta_{g}\right) \rightarrow \mathrm{NO}\left(X^{2} \prod_{r}\right)+\mathrm{O}\left({ }^{3} P\right) \\
& \mathrm{N}\left({ }^{4} S\right)+\mathrm{O}_{2}\left(a^{1} \Delta_{g}\right) \rightarrow \mathrm{N}\left({ }^{4} S\right)+\mathrm{O}_{2}\left(X^{3} \Sigma_{g}^{-}\right) \\
& \mathrm{N}\left({ }^{4} S\right)+\mathrm{O}_{2}\left(b^{1} \Sigma_{g}^{+}\right) \rightarrow \mathrm{N}\left({ }^{2} D\right)+\mathrm{O}_{2}\left(X^{3} \Sigma_{g}^{\bar{g}}\right)
\end{aligned}
$$

\section{4. Заключение}

В ходе теоретического исследования системы $\mathrm{N}\left({ }^{4} S\right)+\mathrm{O}_{2}\left(\left\{X^{3} \Sigma_{g}^{-}, a^{1} \Delta_{g}, b^{1} \Sigma_{g}^{+}\right\}\right)$в рамках расширенной многоконфигурационной квазивырожденной теории возмущения второго порядка была проведена серия квантово-химических расчетов, в результате которых были выявлены возможные пути реакции, а также были найдены точки межсистемных переходов.

Впервые была показана теоретическая возможность неадиабатических переходов $\mathrm{N}\left({ }^{4} S\right)+\mathrm{O}_{2}\left(a^{1} \Delta_{g}\right) \rightarrow \mathrm{N}\left({ }^{4} S\right)+\mathrm{O}_{2}\left(X^{3} \Sigma_{g}^{-}\right)$, вследствие чего при оценке константы скорости реакции $\mathrm{N}\left({ }^{4} S\right)+\mathrm{O}_{2}\left(a^{1} \Delta_{g}\right) \rightarrow \mathrm{NO}\left(X^{2} \Pi_{r}\right)+\mathrm{O}\left({ }^{3} P\right)$ необходимо учитывать вероятность данного перехода. Полученное значение активационного барьера данной реакции превышает энергию активации реакции $\mathrm{N}\left({ }^{4} S\right)+\mathrm{O}_{2}\left(X^{3} \Sigma_{g}^{-}\right) \rightarrow \mathrm{NO}\left(X^{2} \Pi_{r}\right)+\mathrm{O}\left({ }^{3} P\right)$ примерно в 2.3 раза, а также оказалось меньше примерно в 2.2 раза по сравнению с результатом одной из прошлых работ [9]. Это связано с тем, что в рамках методологии настоящей работы учитывалась динамическая энергия корреляции, тогда как в методологии работы [9] данные поправки не были учтены.

Также впервые был выявлен возможный реакционный канал $\mathrm{N}\left({ }^{4} S\right)+\mathrm{O}_{2}\left(b^{1} \Sigma_{g}^{+}\right) \rightarrow$ $\rightarrow \mathrm{NO}\left(X^{2} \prod_{r}\right)+\mathrm{O}\left({ }^{3} P\right)$, однако оценка константы скорости реакции данного канала осложняется множеством пересечений с различными термами системы $\mathrm{N}+\mathrm{O}_{2}$. Несмотря на это, в рамках неадиабатической теории переходного состояния можно провести оценку константы скорости процесса электронного обмена $\mathrm{N}\left({ }^{4} S\right)+\mathrm{O}_{2}\left(b^{1} \Sigma_{g}^{+}\right) \rightarrow \mathrm{N}\left({ }^{2} D\right)+\mathrm{O}_{2}\left(X^{3} \Sigma_{g}^{-}\right)$. 
На основе полученных в ходе данной работы результатов в дальнейшем планируются расчеты констант скорости выявленных процессов в рамках вариационной теории переходного состояния и с учетом вероятности неадибатических переходов.

\section{Благодарности и ссылки на гранты}

Работа была выполнена при поддержке Российского Фонда Фундаментальных Исследований (проекты РФФИ №18-31-00140 и №17-01-00810).

\section{Литература}

1. A. Starikovskiy, N. Aleksandrov Plasma-assisted ignition and combustion // Prog. Energy Combust. Sci. 2013, V. 39, Pp. 61-110.

2. S.M. Starikovskaia Plasma-assisted ignition and combustion: nanosecond discharges and development of kinetic mechanisms // J. Phys. D: Appl. Phys. 2014, V. 47, N. 35.

3. I.V. Adamovich, W.R. Lempert Challenges in understanding and predictive model development of plasma-assisted combustion // Plasma Phys. Controlled Fusion 2015, V. 57, N. 1.

4. Y. Ju, W. Sun Plasma assisted combustion: Dynamics and chemistry // Prog. Energy Combust. Sci. 2015, V. 48, Pp. 21-83.

5. Y. Ju, J.K. Lefkowitz, C.B. Reuter, S.H. Won, X. Yang, S. Yang, W. Sun, Z. Jiang, Q. Chen Plasma Assisted Low Temperature Combustion // Plasma Chem. Plasma Process. 2016, V. 36, Pp. 85-105.

6. M. Castela, B. Fiorina, A. Coussement, O. Gicquel, N. Darabiha, C.O. Laux Modelling the impact of non-equilibrium discharges on reactive mixtures for simulations of plasma-assisted ignition in turbulent flows // Combust. Flame 2016, V. 166, Pp. 133-147.

7. Q. Chen, X. Yang, J. Sun, X. Zhang, X. Mao, Y. Ju, B.E. Koel Pyrolysis and Oxidation of Methane in a RF Plasma Reactor // Plasma Chem. Plasma Process. 2017, V. 37, Pp. 1551-1571.

8. E.A. Filimonova, A.N. Bocharov, A.S. Dobrovolskaya, V.A. Bityurin Influence of Nanoseconds Pulsed Discharges on the Composition of Intermediate and Final Combustion Products in the HCCI Engine // Plasma Chem. Plasma Process. 2019, V. 39, Pp. 683-694.

9. A.M. Starik, P.S. Kuleshov, A.S. Sharipov, V.A. Strelnikova, N.S. Titova On the influence of singlet oxygen molecules on the $\mathrm{NO}_{x}$ formation in methane-air laminar flame // Proc. Combust. Inst. 2012, V. 34, N. 2, Pp. 3277-3285.

10. P.J. Bruggeman, F. Iza, R. Brandenburg Foundations of atmospheric pressure non-equilibrium plasmas // Plasma Sources Sci. Technol. 2017, V. 26, N. 12.

11. A.M. Starik, B.I. Loukhovitski, A.S. Sharipov, N.S. Titova Physics and chemistry of the influence of excited molecules on combustion enhancement // Phil. Trans. R. Soc. A 2015, V. 373, P. 20140341.

12. V.V. Smirnov, O.M. Stelmakh, V.I. Fabelinsky, D.N. Kozlov, A.M. Starik, N.S. Titova On the influence of electronically excited oxygen molecules on combustion of hydrogen-oxygen mixture // J. Phys. D: Appl. Phys. 2008, V. 41, N. 19.

13. N.P. Vagin, I.V. Kochetov, A.P. Napartovich, N.N. Yuryshev Influence of chemically produced singlet delta oxygen molecules on thermal ignition of $\mathrm{O}_{2}-\mathrm{H}_{2}$ mixtures // J. Phys. D: Appl. Phys. 2016, V. 49, N. 5.

14. A.M. Starik, V.E. Kozlov, N.S. Titova Modeling Study of the Possibility of HCCI Combustion Improvement via Photochemical Activation of Oxygen Molecules // Energy Fuels 2014, V. 28, Pp. 2170 2178.

15. A.M. Starik, A.V. Pelevkin, N.S. Titova Modeling study of the acceleration of ignition in ethane-air and natural gas-air mixtures via photochemical excitation of oxygen molecules // Combust. Flame 2017, V. 176, Pp. 81-93.

16. A. Tropina, Y. Wu, C. Limbach, R.B. Miles Aero-optical effects in non-equilibrium air // 2018 Plasmadynamics and Lasers Conference. 2018, P. 3904, DOI 10.2514/6.2018-3904. 
17. I.N. Kadochnikov, I.V. Arsentiev. Kinetics of nonequilibrium processes in air plasma formed behind shock waves: state-to-state consideration // J. Phys. D: Appl. Phys. 2018, V. 51, P. 374001 (22 p.).

18. A.A. Granovsky Extended multi-configuration quasi-degenerate perturbation theory: The new approach to multi-state multi-reference perturbation theory // J. Chem. Phys. 2011, V. 134, N. 21.

19. B.O. Roos The complete active space self-consistent field method and its applications in electronic structure calculations//Advances in Chemical Physics: Ab Initio Methods in Quantum Chemistry Part 2. 1987, V. 69, Pp. 399-445.

20. R.A. Kendall, T.H. Dunning Jr., R.J. Harrison Electron affinities of the first-row atoms revisited. Systematic basis sets and wave functions // J. Chem. Phys. 1992, V. 96, N. 9, Pp. 6796-6806.

21. A.A. Granovsky. Firefly V. 8.2.0. URL http://classic.chem.msu.su/gran/firefly/index.html.

22. M.W. Schmidt, K.K. Baldridge, J.A. Boatz, S.T. Elbert, M.S. Gordon, J.H. Jensen, S. Koseki, N. Matsunaga, K.A. Nguyen, S. Su, T.L. Windus, M. Dupuis, J.A. Montgomery General atomic and molecular electronic structure system // J. Comput. Chem. 1993, V 14, N. 11, Pp. 1347-1363.

23. C. Gonzalez, B.H. Schlegel An improved algorithm for reaction path following // J. Chem. Phys. 1989, V. 90, N. 4, Pp. 2154-2161.

24. S.Matsika Conical intersections in molecular systems // Reviews in computational chemistry. 2007, V. 23, Chap. 2, Pp. 83-124.

25. K.P. Huber, G. Herzberg Molecular Spectra and Molecular Structure. Vol. 4. Constants of Diatomic Molecules // Van Nostrand Reinhold, New York, 1979.

26. A. Fernandez, A. Goumri, A. Fontijn Kinetics of the reactions of $\mathrm{N}\left({ }^{4} S\right)$ atoms with $\mathrm{O}_{2}$ and $\mathrm{CO}_{2}$ over wide temperatures ranges // J. Phys. Chem. A 1998, V. 102, N. 1, Pp. 168-172.

27. B. Ruscic, D.H. Bross. Active thermochemical tables (ATcT) values based on ver. 1.122 of the thermochemical network (2016). URL https://atct.anl.gov/.

Статья поступила в редакцию 25 октября 2019 г. 